\title{
OPE, Heavy-Quark Mass, and Heavy-Meson Decay Constants from QCD Sum Rules
}

\author{
Wolfgang Lucha \\ HEPHY, Austrian Academy of Sciences, Nikolsdorfergasse 18, A-1050 Vienna, Austria \\ E-mail: wolfgang.lucha@oeaw.ac.at \\ Dmitri Melikhov* \\ HEPHY, Austrian Academy of Sciences, Nikolsdorfergasse 18, A-1050 Vienna, Austria \\ Faculty of Physics, University of Vienna, Boltzmanngasse 5, A-1090 Vienna, Austria \\ SINP, Moscow State University, 119991, Moscow, Russia \\ E-mail: dmitri_melikhov@gmx.de
}

\section{Silvano Simula}

INFN, Sezione di Roma III, Via della Vasca Navale 84, I-00146 Roma, Italy

E-mail: simula@roma3.infn.it

\begin{abstract}
We present a sum-rule extraction of the decay constants of heavy-light mesons from the two-point correlator of pseudoscalar currents [1]. To this end, we compare the perturbative expansions for the correlator and the decay constant performed in terms of either the pole mass or the running $\overline{\mathrm{MS}}$ mass of the heavy quark. The perturbative expansion expressed in terms of the pole mass exhibits no sign of convergence whereas reorganizing this very expansion in terms of the $\overline{\mathrm{MS}}$ mass yields a rather clear hierarchy of the perturbative contributions. Accordingly, the decay constants extracted from the pole-mass correlator turn out to be considerably smaller than those extracted from its $\overline{\mathrm{MS}}$-mass counterpart. Then, making use of the OPE in terms of the $\overline{\mathrm{MS}}$ mass we derive the decay constants of heavy mesons with emphasis on acquiring control over the uncertainties in the decay constants, related both to the input QCD parameters and to the limited accuracy of the method of sum rules. Gaining this control has become possible due to the application of our novel procedure for extracting hadron observables based on dual thresholds which depend on the Borel parameter.
\end{abstract}

The XIXth International Workshop on High Energy Physics and Quantum Field Theory

8-15 September 2010

Golitsyno, Moscow, Russia

\footnotetext{
* Speaker.
} 


\section{Introduction: QCD Sum Rules, Quark-Hadron Duality, and Effective Thresholds}

The extraction of the decay constant of any ground-state heavy pseudoscalar meson within the method of QCD sum rules [2] poses, for the following well-known reasons, a challenging problem:

First, one has to derive a reliable operator product expansion (OPE) for the correlation function

$$
\Pi\left(p^{2}\right) \equiv \mathrm{i} \int \mathrm{d}^{4} x \exp (\mathrm{i} p x)\left\langle 0\left|T\left(j_{5}(x) j_{5}^{\dagger}(0)\right)\right| 0\right\rangle
$$

of two pseudoscalar heavy-light currents $j_{5}(x) \equiv\left(m_{Q}+m\right) \bar{q}(x) \mathrm{i} \gamma_{5} Q(x)$, with quark masses $m_{Q}, m$.

Second, the knowledge of the truncated OPE for the correlator - even if the parameters of this OPE are known precisely - allows to extract the characteristics of the bound state with only limited accuracy which reflects the intrinsic uncertainties of the method of QCD sum rules. Gaining control over these uncertainties is a very subtle problem [3].

Let us briefly recall the essential features of the sum-rule computation of the decay constants. The quark-hadron duality assumption yields a relation between ground-state contribution and OPE for the Borel-transformed correlator with a cut applied at some effective continuum threshold $s_{\text {eff: }}$ :

$$
f_{Q}^{2} M_{Q}^{4} \exp \left(-M_{Q}^{2} \tau\right)=\Pi_{\text {dual }}\left(\tau, s_{\text {eff }}\right) \equiv \int_{\left(m_{Q}+m\right)^{2}}^{s_{\text {eff }}} \mathrm{d} s \exp (-s \tau) \rho_{\text {pert }}(s)+\Pi_{\text {power }}(\tau)
$$

where the perturbative spectral density is obtained as expansion in powers of the strong coupling $\alpha_{\mathrm{s}}$ :

$$
\rho_{\text {pert }}(s)=\rho^{(0)}(s)+\frac{\alpha_{\mathrm{s}}}{\pi} \rho^{(1)}(s)+\left(\frac{\alpha_{\mathrm{s}}}{\pi}\right)^{2} \rho^{(2)}(s)+\cdots .
$$

Evidently, in order to extract the decay constant one has to fix the effective continuum threshold $s_{\text {eff }}$.

A crucial albeit rather obvious observation is that $s_{\text {eff }}$ has to be a function of $\tau$. Otherwise the 1.h.s. and the r.h.s. of (1.1) exhibit a different $\tau$-behavior. The exact effective continuum threshold, corresponding to exact values of hadron mass and decay constant on the 1.h.s. of (1.1), is, of course, not known. Therefore, the extraction of hadron parameters from the sum rule consists in attempting (i) to find a reasonable approximation to the exact threshold and (ii) to control the accuracy of this approximation. In a series of publications [4] we have formulated all the corresponding procedures.

Let us introduce the dual invariant mass $M_{\text {dual }}$ and the dual decay constant $f_{\text {dual }}$ by the relations

$$
M_{\text {dual }}^{2}(\tau) \equiv-\frac{\mathrm{d}}{\mathrm{d} \tau} \log \Pi_{\text {dual }}\left(\tau, s_{\mathrm{eff}}(\tau)\right), \quad f_{\text {dual }}^{2}(\tau) \equiv M_{Q}^{-4} \exp \left(M_{Q}^{2} \tau\right) \Pi_{\text {dual }}\left(\tau, s_{\text {eff }}(\tau)\right) .
$$

If the ground-state mass is known, any deviation of the dual mass from the actual ground-state mass yields an indication of the amount of excited-state contribution picked up by our dual correlator. Assuming a particular behavior of the effective threshold with $\tau$ and requiring the least deviation of the dual mass in (1.2) from the actual mass $M_{Q}$ in the $\tau$-window yields a variational solution for the effective threshold $s_{\text {eff }}(\tau)$. As soon as the latter has been fixed we get the decay constant from (1.2). The standard assumption for the effective threshold is a $\tau$-independent constant. In addition to this approximation, we have considered polynomials in $\tau$. Reproducing the actual mass considerably improves for $\tau$-dependent thresholds. This means that a dual correlator with $\tau$-dependent threshold isolates the ground-state contribution much better and is less contaminated by the excited states than 
a dual correlator with standard $\tau$-independent threshold. As consequence, the accuracy of extracted hadron observables is significantly increased. Recent experience from potential models reveals that the band of values obtained from the linear, quadratic, and cubic Ansätze for the effective threshold encompasses the true value of the decay constant [4]. Moreover, we managed to demonstrate that the extraction procedures in quantum mechanics and QCD are even quantitatively rather similar [5]. This contribution reports our recent findings $[1,6]$ for pseudoscalar-heavy-meson decay constants.

\section{OPE and Heavy-Quark Mass}

For heavy-light correlators and decay constants it makes a big difference which scheme for the heavy-quark mass is used. We adopt the OPE for this correlator to three-loop accuracy [7], which was obtained in terms of the pole mass of the heavy quark. The pole-mass scheme is the standard one; it has been used for a long time since the pioneering work of Ref. [8]. An alternative is to reorganize the perturbative expansion in terms of the running $\overline{\mathrm{MS}}$ mass [9]. Since the correlator is known to $\alpha_{\mathrm{s}}^{2}$ accuracy, the relation between the pole and the $\overline{\mathrm{MS}}$ mass to the same accuracy is used. Figure 1 depicts the corresponding spectral densities and our sum-rule $f_{B}$ estimates for both cases.
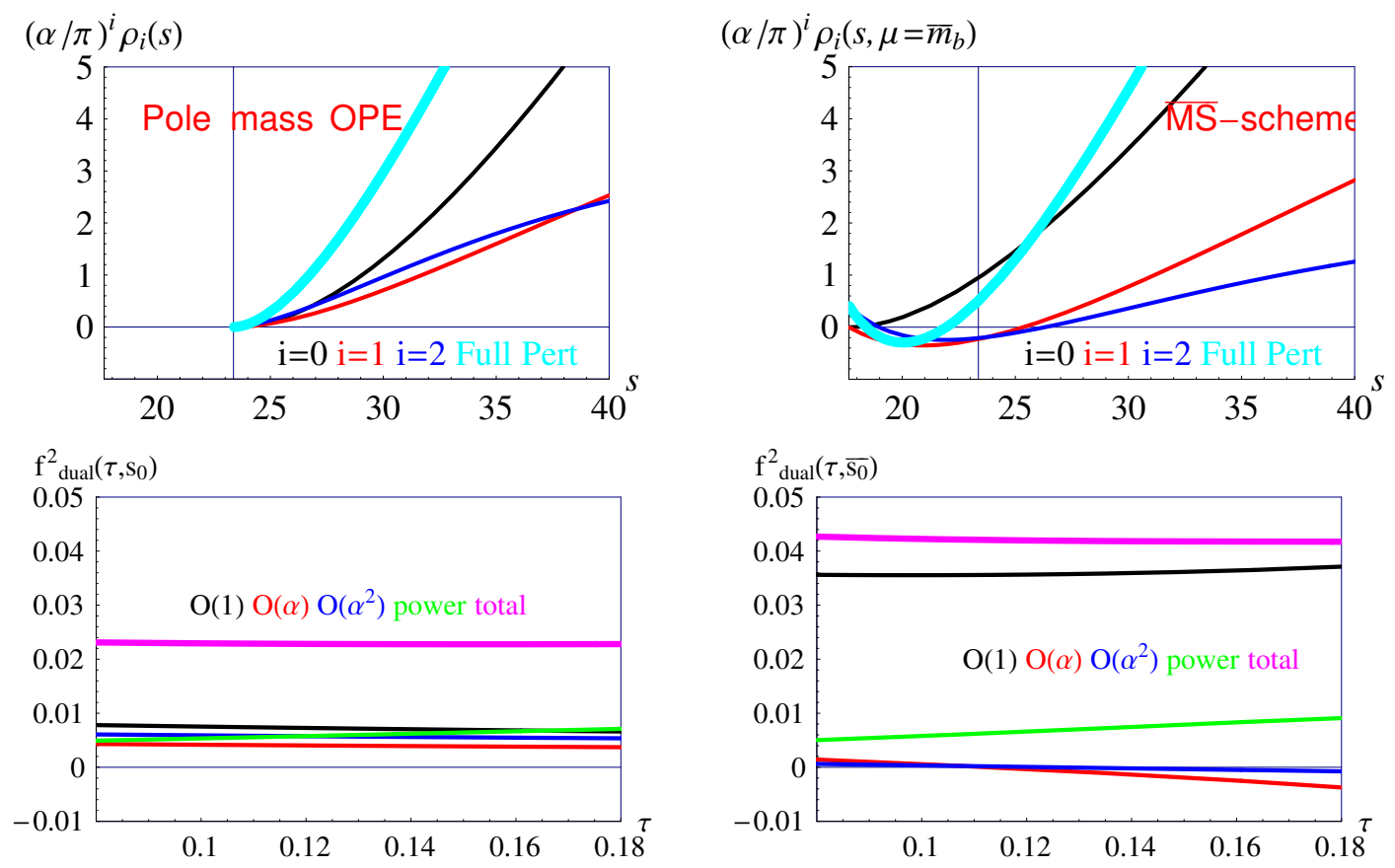

Figure 1: OPE expressed in terms of the pole mass (left) and the $\overline{\mathrm{MS}}$ mass (right) of the $b$ quark: First row: spectral densities. Second row: corresponding sum-rule estimates for $f_{B}$; a constant effective continuum threshold is fixed in each case by requiring maximum stability of the extracted decay constant, thus $s_{0} \neq \bar{s}_{0}$.

Several important lessons may be learnt from the instructive plots in Fig. 1:

(i) The perturbative expansion for the decay constant in terms of the pole mass exhibits no sign of convergence; each of the terms - LO, NLO, NNLO — gives a positive contribution of similar size. Thus, there is no reason to expect that higher orders give smaller contributions. As a consequence, the decay constant extracted from the pole-mass OPE considerably underestimates the actual value. 
(ii) Reorganizing the perturbative series in terms of the $\overline{\mathrm{MS}}$ mass of the heavy quark leads to a clear hierarchy of the perturbative contributions [9]. Notice, however, that also in this case the situation is not perfect: the full spectral density — which is a positive-definite function — is negative at small values of $s$. This is an artifact of the truncation and indicates that the contributions of higher-order terms are non-negligible.

(iii) The absolute value of the decay constant obtained from the pole-mass OPE proves to be almost $50 \%$ smaller than in the case of the $\overline{\mathrm{MS}}$ scheme. Let us emphasize that, nevertheless, in both cases the decay constant exhibits perfect stability over a wide range of the Borel parameter $\tau$. Thus, mere Borel stability is, by far, not sufficient to guarantee the reliability of the sum-rule extraction of bound-state parameters. We have pointed out this fact already more than once [3]. Unfortunately, some authors still adhere to the idea that Borel stability is a "proof" of the reliability of their results.

Because of the obvious problems with the pole-mass OPE for the correlator, following [9] we make use of the OPE formulated in terms of the $\overline{\mathrm{MS}}$ mass for the extraction of the decay constants.

\section{Decay Constants of $D$ and $D_{s}$ Mesons}

The application of our modified extraction prescriptions relying on the $\tau$-dependent effective threshold leads to the following results for charmed mesons (a detailed analysis can be found in [1]):

$$
\begin{aligned}
f_{D} & =\left(206.2 \pm 7.3_{(\mathrm{OPE})} \pm 5.1_{(\text {syst })}\right) \mathrm{MeV} \\
f_{D_{s}} & =\left(245.3 \pm 15.7_{(\mathrm{OPE})} \pm 4.5_{(\text {syst })}\right) \mathrm{MeV}
\end{aligned}
$$

The OPE-related error is obtained by bootstrapping, allowing for a variation of all QCD parameters (i.e., quark masses, $\alpha_{\mathrm{s}}$, condensates) in the relevant ranges. One observes a perfect agreement of our predictions with the respective lattice results (Fig. 2). It should be emphasized that our $\tau$-dependent
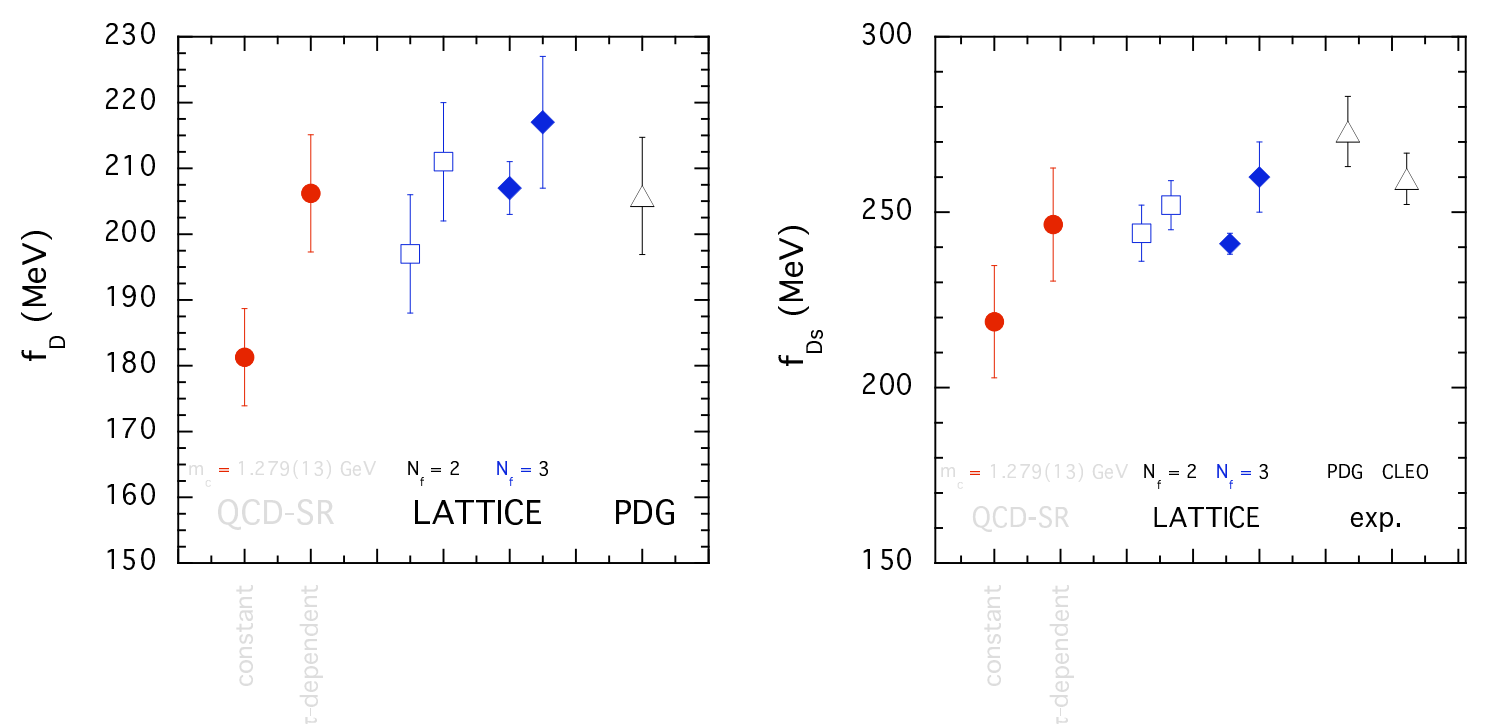

Figure 2: Comparison of our findings for $f_{D}$ and $f_{D_{s}}$ with lattice results and experiment; for details, see [1]. 
threshold is a crucial ingredient for a successful extraction of the decay constants from the sum rule. Obviously, the standard $\tau$-independent naïve approximation yields a much lower value for $f_{D}$ which lies rather far from the empirical data deduced from experiment and from the lattice computations.

Let us emphasize the following: In quantum-mechanical potential models [4] we succeeded to show that taking into account the $\tau$-dependence of the effective threshold considerably improves the accuracy of the duality approximation and the quality of the sum-rule estimates. The investigation of the decay constant of the $D$ meson clearly demonstrates that also in QCD the $\tau$-dependent threshold leads to a much better accuracy of the duality approximation (see Figs. 1, 3, 5, and 8 of Ref. [1]) and to a discernible improvement of the accuracy of the extracted decay constant. This perfectly confirms our observation [5] that the extraction procedures in QCD and in quantum mechanics are very similar to each other - both qualitatively and quantitatively. Moreover, our $D$ meson analysis gives strong arguments that our algorithm provides quite realistic systematic errors.

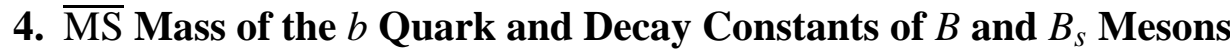

The values of the beauty-meson decay constants extracted from QCD sum rules are extremely sensitive to the chosen $b$ mass $\bar{m}_{b}\left(\bar{m}_{b}\right)$. For instance, the range $\bar{m}_{b}\left(\bar{m}_{b}\right)=(4.163 \pm 0.016) \mathrm{GeV}$ [10] yields results for the decay constants that are barely compatible with the lattice calculations (Fig. 3). Requiring our sum-rule result for $f_{B}$ to match the average of the lattice determinations entails the rather precise value of the $b$-quark mass

$$
\bar{m}_{b}\left(\bar{m}_{b}\right)=(4.245 \pm 0.025) \mathrm{GeV} .
$$

Our sum-rule estimates for $f_{B}$ and $f_{B_{s}}$ corresponding to this value of the $b$-quark mass thus become

$$
\begin{aligned}
f_{B} & =\left(193.4 \pm 12.3_{(\mathrm{OPE})} \pm 4.3_{(\mathrm{syst})}\right) \mathrm{MeV}, \\
f_{B_{s}} & =\left(232.5 \pm 18.6_{(\mathrm{OPE})} \pm 2.4_{(\mathrm{syst})}\right) \mathrm{MeV} .
\end{aligned}
$$
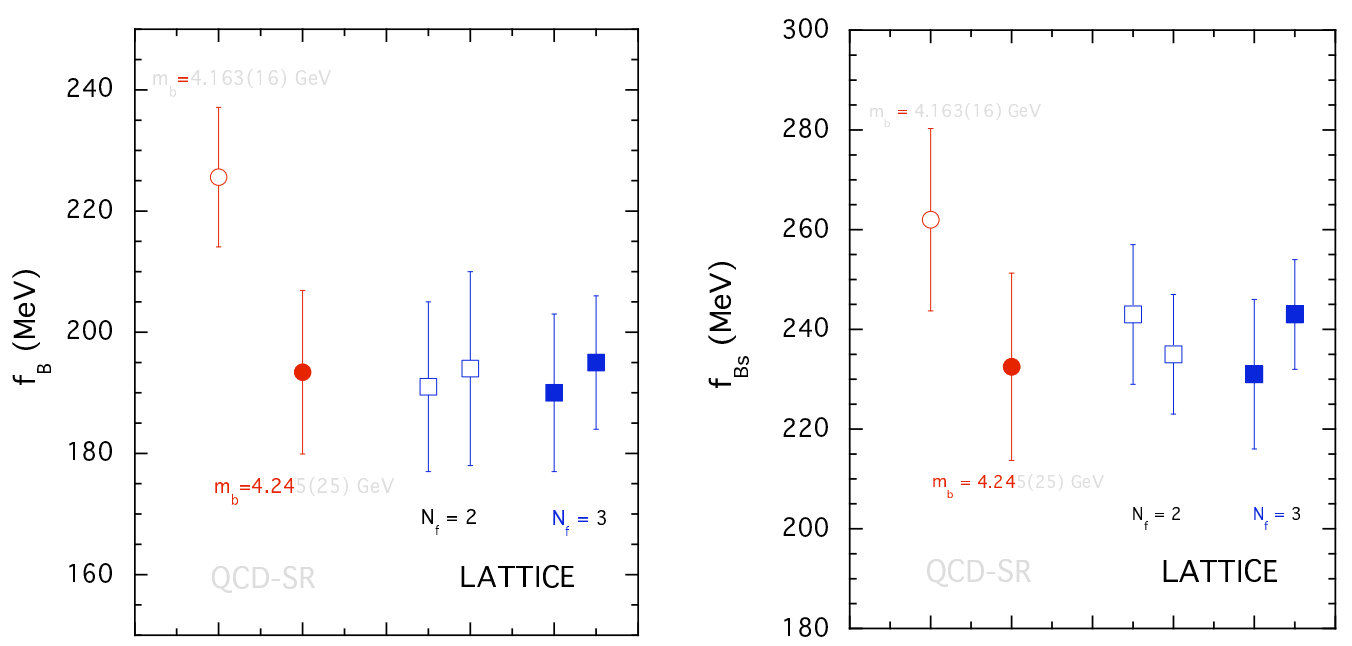

Figure 3: Comparison of our results for $f_{B}$ and $f_{B_{s}}$ with lattice results; for a detailed list of references, cf. [1]. 


\section{Summary and Conclusions}

The main insights gained in our comprehensive inspection $[1,3,4,5,6]$ of the QCD sum-rule approach and its potential improvement by reasonable modifications can be summarized as follows:

1. The $\tau$-dependence of the effective continuum threshold emerges naturally when one attempts to make the duality relation exact: this dependence is obvious from (1.1). We would like to emphasize the following two points: (a) In principle, the $\tau$-dependence cannot be in conflict with any property of quantum field theories. (b) Many examples, in particular, the analysis of the decay constants of $D$ mesons presented here, show that it indeed improves visibly the quality of the sum-rule predictions.

2. Our analysis of charmed mesons clearly demonstrates that the use of Borel-parameter-dependent thresholds leads to two essential improvements: (i) The accuracy of decay constants extracted from sum rules is considerably improved. (ii) It has become possible to obtain realistic systematic errors and to reduce their values to the level of a few percent. The application of our prescription brings the QCD sum-rule results into perfect agreement with the findings of both lattice QCD and experiment.

3. The beauty-meson decay constants prove to be extremely sensitive to the chosen value of $\bar{m}_{b}\left(\bar{m}_{b}\right)$; matching the results from QCD sum rules for $f_{B}$ to the average of the lattice evaluations allows us to provide a rather accurate estimate of the $b$-quark mass. Our $\bar{m}_{b}\left(\bar{m}_{b}\right)$ value is in good agreement with several lattice results but, interestingly, does not overlap with the recent accurate determination presented in Ref. [10] (for details, see Ref. [1]). Of course, this intriguing puzzle should be clarified.

Acknowledgments. D.M. was supported by the Austrian Science Fund (FWF), project no. P20573.

\section{References}

[1] W. Lucha, D. Melikhov, and S. Simula, arXiv:1008.2698 [hep-ph].

[2] M. Shifman, A. Vainshtein, and V. Zakharov, Nucl. Phys. B 147, 385 (1979).

[3] W. Lucha, D. Melikhov, and S. Simula, Phys. Rev. D 76, 036002 (2007); Phys. Lett. B 657, 148 (2007); Phys. Atom. Nucl. 71, 1461 (2008); Phys. Lett. B 671, 445 (2009); D. Melikhov, Phys. Lett. B 671, 450 (2009).

[4] W. Lucha, D. Melikhov, and S. Simula, Phys. Rev. D 79, 096011 (2009); J. Phys. G: Nucl. Part. Phys. 37, 035003 (2010); W. Lucha, D. Melikhov, H. Sazdjian, and S. Simula, Phys. Rev. D 80, 114028 (2009).

[5] W. Lucha, D. Melikhov, and S. Simula, Phys. Lett. B 687, 48 (2010); Phys. Atom. Nucl. 73, 1770 (2010).

[6] W. Lucha, D. Melikhov, and S. Simula, arXiv:1008.2951 [hep-ph]; arXiv:1008.3129 [hep-ph]; arXiv:1011.3372 [hep-ph].

[7] K. G. Chetyrkin and M. Steinhauser, Phys. Lett. B 502, 104 (2001); Eur. Phys. J. C 21, 319 (2001).

[8] T. M. Aliev and V. L. Eletsky, Yad. Fiz. 38, 1537 (1983).

[9] M. Jamin and B. O. Lange, Phys. Rev. D 65, 056005 (2002).

[10] K. G. Chetyrkin et al., Phys. Rev. D 80, 074010 (2009). 